\title{
On the microwave oscillations from active region NOAA 0139
}

\author{
G.B. Gelfreikh ${ }^{1}$, L.I. Tsvetkov ${ }^{2}$, Y.F. Yurovsky ${ }^{2}$, Y.T. Tsap ${ }^{2}$, B.V.

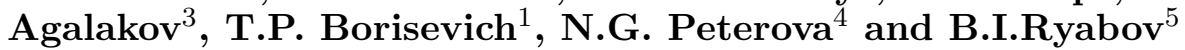 \\ ${ }^{1}$ Central astronomical observatory at Pulkovo of RAS, St.-Petersburg, Russia \\ ${ }^{2}$ Crimean Astrophysical Observatory, Ukraine \\ ${ }^{3}$ Institute of Solar-Terrestrial Physics, Irkutsk, Russia \\ ${ }^{4}$ St.-Petersburg branch of the Special Astrophysical Observatory, St.-Petersburg, Russia \\ ${ }^{5}$ Ventspils International Radioastronomical Centre, Riga, Latviya
}

\begin{abstract}
This investigation is devoted to the study of time variations and spatial peculiarities of radio local radio sources associated with the solar active regions (AR) on the basis of microwave observations in September-October 2002. During this period the most interesting results have been obtained for the AR NOAA 0139. So, in this paper an analysis of this radio source is presented. The observations were made with the radio telescopes RT-22 (Crimea), RATAN-600, the Siberian Solar Radio Telescope (SSRT) and Nobeyama. Observations with the RT-22 (wavelengths $2.0,2.3,3.5 \mathrm{~cm}$ ) were carried out by the quasi-zero method with the flux sensitivity of 0.1 s.f.u and the temporal resolution of about $0.1 \mathrm{~s}$. The typical length of realization was about 6.5 hours. The spatial resolution of few arc minutes allowed to limit the analysis by the particular AR but was not enough to distinguish any details. Nevertheless spectral analysis of the AR made using observations with high one-dimensional resolution of the RATAN-600 shows that at short $\mathrm{cm}$ wavelengths for this AR at the period of the RT-22 observations dominated thermal cyclotron emission of above the largest sunspot of the group. This result was also confirmed by radio maps of the Nobeyama at wavelength $1.76 \mathrm{~cm}$.
\end{abstract}

\section{Spatial peculiarities of the AR NOAA 0139}

At the photosphere level NOAA 0139 demonstrates the large sunspot group with the area of about $6 \cdot 10^{-4}$ of the solar semi-sphere. The spot of $\mathrm{N}$-polarity magnetic field was dominating. According to the SOHO MDI data this magnetic field has vastly increased from 1.2 KG to $1.8 \mathrm{KG}$ at the period of October (06-07). The magnetic field dynamic at the photosphere level was reflected in the corona structure above this AR. The set of RATAN-scans and SSRT-maps at the period (05-09).10 show that the most significant changes of the radio source structure took place at the short wavelengths $(\lambda \sim 2 \mathrm{~cm})$ and relate to the brightest detail associated with the main sunspot. The flux density and the brightness temperature at 08.10 drastic drops whereas no changes of these characteristics at the longer wavelengths are in evidence here. The flux density and polarization degree spectra show that the parameters of this detail at the short wavelengths correspond to the thermal cyclotron emission. Taking into account the model of a cyclotron source it follows that the increase in the photospheric magnetic field was accompanied by the essential reconstruction of the chromosphere-corona transition region. We found that the 3 d gyro level at the wavelength $3 \mathrm{~cm}(1200 \mathrm{Gs})$ was all time situated in corona while at the wavelength $2 \mathrm{~cm}$ and even $1.76 \mathrm{~cm}(2000 \mathrm{Gs})$ it was moved from the corona to the cooler chromosphere level. 


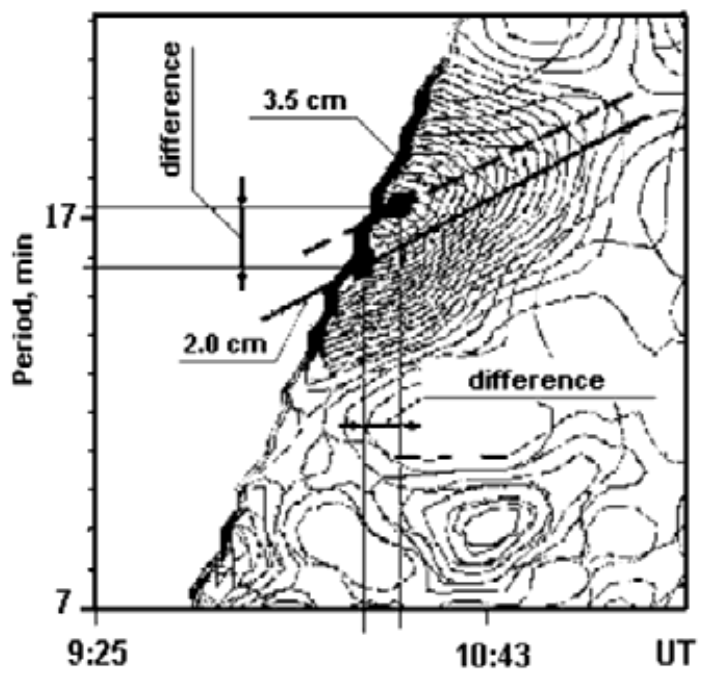

Figure 1. Fragment of wavelet spectrum of AR NOAA 0139 radio emission oscillations at the wavelength $2 \mathrm{~cm}$ combined with that at the wavelength $3.5 \mathrm{~cm}$ according to RT-22 data.

\section{Time variation of the AR NOAA 0139 radio emission}

To study unstable spectral variations of the radio emission observed with the RT-22 we used wavelet analysis (Morlet). The microwave oscillations at 07.09 were revealed with characteristic periods of about 50, 20, and $17 \mathrm{~min}$. Amplitudes of these oscillations were increased with the periods. According to the detailed investigations of pulsations by the wavelet analysis (see figure 1 ) oscillations with period $\sim 17$ min were not stable its period increases with increasing of the wavelength and oscillation maximum with time displaced to the longer wavelengths. This phenomenon can be interpreted as a consequence of a disturbance which was propagating from foot points to top of the loop resulting in variation of its resonance parameters.

\section{Results}

The gyroresonance emitting region responsible in our case for the oscillating radio emission is generated in physically very thin layer which itself can not be resonator for any MHD oscillations. So, the observed periods are due to the effect of the resonators outside the region of generation of the radio emission. Possible resonator may be due to the magnetic coronal loops coming from the region of the main sunspot and having similar characteristics, but slightly different oscillation frequencies (e.g., $\sim 20 \mathrm{~min}$ ). Reconstruction of the whole structure of the AR in the corona found from the above observations certainly resulted in reconstruction of the coronal loop structures. This also should result in variations of the observed periods of oscillations and the other parameters of those.

\section{Acknowledgements}

The work is supported by the State Research Programs "Astronomy", the "Integration" (I0208.1173), the Scientific School grant 477.2003.2, INTAS 00-0543, contract 16KI, contract OFN-18, contract of Presidium of RAS No 7/2004 and grants of RFBR 02-02-16548, 03-02-17357, 03-02-17528. 